\title{
Construction of Thirty Six Points Second Order Rotatable Design in Three Dimensions with a Practical Hypothetical Case Study
}

\author{
Nyakundi Omwando Cornelious \\ Department of Statistics and Computer Science, Moi University, Eldoret,
}

Kenya

\begin{abstract}
In this study, a new thirty six points second order rotatable design is constructed using trigonometric functions and a practical hypothetical example is given by converting coded levels to natural levels. This design permits a response surface to be fitted easily and provides spherical information contours besides the economic use of scarce resources in relevant production processes.
\end{abstract}

Key words: Response Surface, Rotatable Designs, Second Order.

\section{INTRODUCTION}

In many experimental situations, the researchers are concerned with explaining certain aspects of a functional relationshipy $=$ $f\left(x_{1}\right), f\left(x_{2}\right), \ldots, f\left(x_{k}\right)+e$ where $y$ is the response, $x_{1}, x_{2}, \ldots, x_{k}$ are the levels of $k$ quantitative variables or factors and $e$ is the random error. Response surface methodology is a statistical technique which is very useful in analysis of scientific experiments where several independent variables influence a dependent variable. The response is assumed to be a random variable while the independent variables are assumed to be continuous and are controlled by the experimenter. For example if a farmer wishes to find the Potash $\left(x_{1}\right)$, Nitrogen $\left(x_{2}\right)$ and Phosphate $\left(x_{3}\right)$ ferlizer levels that maximizes the yield, the observed response $y$ may be written as a function of the levels of potash, nitrogen and phosphate fertilizers as $y=f\left(x_{1}, x_{2}, x_{3}\right)+e$. The concept of rotatability which is very important in response surface methodology was introduced by Box and Hunter [3]. They developed second order rotatable designs through geometrical configurations. Bose and Draper [9] point out that the technique of fitting a response surface is one widely used to aid in the statistical analysis of experimental work in which the response of the product depends in some unknown fashion, on one or more controllable variables. Mutiso [8] constructed specific optimal second order rotatable designs in three dimensions. Koske et al. [6, 7] and Keny et al [10] constructed optimal second order rotatable designs and gave practical hypothetical examples. Cheruiyot[1] evaluated the efficiencies of the six second order rotatable designs in three dimensions that were constructed by Mutiso[8]. Cornelious[2, 3, 4 and 5]Constructed optimal sequential third order rotatable designs, and a second order rotatable design of 39 points using trigonometric functions, with a practical hypothetical example. The current study gives yet another new second order rotatable design in three dimensions of thirty six points constructed using trigonometric functions with a practical hypothetical example.

\section{MOMENTS AND NON-SINGULARITY CONDITIONS FOR SECOND ORDER ROTATABILITY}

Suppose we want to use the second order response surface design to fit the surface,

$y_{u}=\beta_{o}+\sum_{u=1}^{N} \beta_{i} x_{i u}+\sum_{u+1}^{N} \beta_{i i} x_{i u}^{2}+\sum_{u=1}^{N} \beta_{i j} x_{i u} x_{j u}+e_{u}$

Where $x_{i}$ denotes the level of the $i^{t h}$ factor $(i=1,2, \ldots k)$ in the $u^{t h}$ run $(u=1,2, \ldots, N)$ of the experiment and $e_{u}$ is the uncorrelated random error with mean zero and variance $\sigma^{2}$ 
A design is said to be a second order rotatable design if the variance of the estimate $y_{u}$ with respect to each independent variable $x_{i u}$ is only a function of the distance $d^{2}=\sum_{u=1}^{N} x_{i u}^{2}$ of the point $\left(x_{1}, x_{2}, \ldots, x_{k}\right)$ from the origin (centre) of the design.

According to Box and Hunter [3], a second order response surface is achieved if the design points satisfy the following conditions,

$\sum_{u=1}^{N} x_{i u}^{2}=\mathrm{N} \lambda_{2}$

$\sum_{u=1}^{N} x_{i u}^{4}=\mathrm{C} \mathrm{N} \lambda_{4}$

$\sum_{u=1}^{N} x_{i u}^{2} x_{j u}^{2}=\mathrm{N} \lambda_{4}$ for $(i \neq j=1,2, \ldots, k)$ and; $\mathrm{C}, \lambda_{2} \& \lambda_{4}$ are constants.

And all other odd order moments are equal to zero.

The non-singularity condition that must be satisfied for a set of points to form a second order rotatable design is given by,

$\frac{\lambda_{4}}{\lambda_{2}^{2}}>\frac{k}{k+2}$

\section{CONSTRUCTION OF A SECOND ORDER ROTATABLE DESIGN USING TRIGONOMETRIC FUNCTIONS TRANSFORMATIONS}

Consider transformations,

$T_{1}=\left[\begin{array}{ccc}\cos \varrho & -\sin \varrho & 0 \\ \sin \varrho & \cos \varrho & 0 \\ 0 & 0 & -1\end{array}\right], \quad T_{2}=\left[\begin{array}{ccc}\cos \frac{\varrho}{2} & -\sin \frac{\varrho}{2} & 0 \\ \sin \frac{\varrho}{2} & \cos \frac{\varrho}{2} & 0 \\ 0 & 0 & -1\end{array}\right]$

Where $\varrho=\frac{2 \pi}{\alpha}$ and $\alpha \geq 4$, given by Bose and Draper [9]

Transformations $T_{1}$ and $T_{2}$ are applied to the points of the form $(r, o, b)$ i.e. points on the plane $\mathrm{y}=\mathrm{o}$ and to all other points obtained from repeated replications of $T_{1}$ and $T_{2}$.

The permutation group $\left(I, W, W^{2}\right)$ generated by,

$\mathrm{W}=\left[\begin{array}{lll}0 & 1 & 0 \\ 0 & 0 & 1 \\ 1 & 0 & 0\end{array}\right]$ is also applied to $(r, o, b)$ to give

$T_{z}(r, o, b), \quad T_{x}(r, o, b)$, and $T_{y}(r, o, b)$,

In all these give $6 \alpha$ points which we denote by $\mathrm{T}(r, o, b)$ and with coordinates.

$\mathrm{S}(r \cos t \varrho, r \sin t \varrho, b)$

$\mathrm{S}\left(r \cos \left(t+\frac{1}{2}\right) \varrho, r \sin \left(t+\frac{1}{2}\right) \varrho, \quad-b\right)$

$\mathrm{S}(b, r \cos t \varrho, r \sin t \varrho)$

$\mathrm{S}\left(-b, r \cos \left(t+\frac{1}{2}\right) \varrho, r \sin \left(t+\frac{1}{2}\right) \varrho,\right)$

$\mathrm{S}(r \cos t \varrho, b, r \sin t \varrho)$

$\mathrm{S}\left(r \cos \left(t+\frac{1}{2}\right) \varrho,-b, r \sin \left(t+\frac{1}{2}\right) \varrho,\right)$

Where $t=0,1,2 \ldots(\alpha-1)$

Applying the conditions in (2), The set $\mathrm{T}(\mathrm{r}, \mathrm{o}, \mathrm{b})$ gives sums of powers and products as, 
$\sum_{u=1}^{N} \mathrm{x}_{\mathrm{iu}}^{2}=\sum_{u=1}^{N} x_{j u}^{2}=\sum_{u=1}^{N} x_{l u}^{2}=2 \alpha\left(r^{2}+b^{2}\right)$

$\sum_{u=1}^{N} x_{i u}^{4}=\sum_{u=1}^{N} x_{j u}^{4}=\sum_{u=1}^{N} x_{l u}^{4}=\frac{\alpha r^{2}\left(3 r^{4}+4 b^{4}\right)}{2}$

$\sum_{u=1}^{N} x_{i u}^{2} x_{j u}^{2}=\sum_{u=1}^{N} x_{j u}^{2} x_{i u}^{2}=\sum_{u=1}^{N} x_{i u}^{2} x_{i u}^{2}=\frac{\alpha r^{2}\left(r^{2}+8 b^{2}\right)}{4}$

And all the other powers and products up to and including order four are zero. Using the properties of sums of powers and products given above, it follows that the excess of the set defined the same way is;

$\sum_{u=1}^{N} x_{i u}^{4}-3 \sum_{u=1}^{N} x_{i u}^{2} x_{j u}^{2}=\frac{\alpha r^{2}\left(3 r^{4}+4 b^{4}\right)}{2}-\frac{\alpha r^{2}\left(r^{2}+8 b^{2}\right)}{4}$

$=\frac{\alpha\left(3 r^{4}-24 r^{2} b^{2}+8 b^{4}\right)}{4}$

The excess of each single point varies in this case and it is necessary to consider the total effect over all points. Since its excess can be made positive or negative according to the choice of $r$ and $b$, it will be possible to combine the set $\mathrm{T}(r, o, b)$ with sets of both positive and negative excesses.

Consider an additional set of 12 points, denoted by $\mathrm{G}(p, p, o)$ whose co-ordinates are given as,

$\mathrm{s}(p, p, o), \mathrm{s}(p, o,-p), \quad \mathrm{s}(o, p, p)$

$\mathrm{s}(-p, p, o) \quad, \mathrm{s}(-p, o, p) \quad, \mathrm{s}(o,-p, p)$

$\mathrm{s}(p,-p, o) \quad, \mathrm{s}(p, o, p) \quad, \mathrm{s}(o, p,-p)$

$\mathrm{s}(-p,-p, o), \mathrm{s}(-p, o,-p) \quad, \mathrm{s}(\mathrm{o},-p,-p)$

The set $\mathrm{G}(p, p, o)$ has sums of powers and products up to order four given by,

$\sum_{u=1}^{N} \mathrm{x}_{\mathrm{iu}}^{2}=\sum_{u=1}^{N} x_{j u}^{2}=\sum_{u=1}^{N} x_{l u}^{2}=8 p^{2}$

$\sum_{u=1}^{N} x_{i u}^{4}=\sum_{u=1}^{N} x_{j u}^{4}=\sum_{u=1}^{N} x_{l u}^{4}=8 p^{4}$

$\sum_{u=1}^{N} x_{i u}^{2} x_{j u}^{2}=\sum_{u=1}^{N} x_{j u}^{2} x_{i u}^{2}=\sum_{u=1}^{N} x_{i u}^{2} x_{i u}^{2}=4 p^{4}$

Using the properties of sums of powers and products given above, it follows that the excess of the set defined the same way is;

$\sum_{u=1}^{N} x_{i u}^{4}-3 \sum_{u=1}^{N} x_{i u}^{2} x_{j u}^{2}=8 p^{4}-12 p^{4}$

$=-4 p^{4}$

\section{CONSTRUCTION OF THIRTY SIX POINTS SECOND ORDER ROTATABLE DESIGN IN THREE DIMENSIONS}

The design is constructed by combining the set $s_{1}=6 \alpha$ points with the 12 point set denoted by $s_{2}=\mathrm{S}(\mathrm{p}, \mathrm{p}, \mathrm{o})$

Combining (4) and (6) gives the following moment conditions.

$\sum_{u=1}^{36} x_{i u}^{2}=2 \alpha\left(r^{2}+b^{2}\right)+8 p^{2}=36 \lambda_{2}$

$\sum_{u=1}^{36} x_{i u}^{4}=\frac{1}{2} \alpha\left(3 \mathrm{r}^{4}+4 \mathrm{~b}^{4}\right)+8 \mathrm{p}^{4}=108 \lambda_{4}$

$\sum_{u=1}^{36} x_{i u}^{2} x_{j u}^{2}=\frac{1}{4} \alpha r^{2}\left(\mathrm{r}^{2}+8 \mathrm{~b}^{2}\right)+4 \mathrm{p}^{4}=36 \lambda_{4}$ 
For $(i \neq j=1,2,3)$ and all other sums and products and powers up to and including order four are zero.

The excess function obtained after combining (5) and (7) is given by,

$\sum_{u=1}^{N} x_{i u}^{4}-3 \sum_{u=1}^{N} x_{i u}^{2} x_{j u}^{2}=3 r^{4}-24 r^{2} b^{2}+8 b^{4}-4 p^{4}=0$

Let $r^{2}=x p^{2}$ and $b^{2}=y p^{2}$

Substituting (10) to (9) gives,

$3 x^{2}-24 x y+8 y^{2}-4=0$

Making $x$ in (11) the subject gives,

$x=\frac{24 y \pm \sqrt{480 y^{2}-48}}{6}$

For the two combined sets to form a rotatable arrangement, the values of $y$ must be chosen in such a way that both $y$ and the corresponding values of $x$ in (12) are real and positive.

Taking the value of $y$ specifically to be 2 , then $x=0.606308995$.

This implies that;

$r^{2}=0.606308995 p^{2}$ and $b^{2}=2 p^{2}$

Substituting (13) to the moment conditions given in (8) gives;

$\lambda_{2}=0.801401998 p^{2}$ and $\lambda_{4}=0.390793181 p^{4}$

Subsisting (14) to (3) for $k=3$, shows that the non singularity condition in (3) is satisfied, thus the thirty six points qualify to be a second order rotatable design in three dimensions.

Table 1: A summary of the moment conditions for the thirty six points second order rotatable design in three dimensions.

\begin{tabular}{|c|c|c|c|}
\hline Set composition of class & $s_{1}=6 \alpha$ & $\begin{array}{l}s_{2} \\
=s(p, p, 0)\end{array}$ & $s_{1}+s_{2}$ \\
\hline Number of points & $\begin{array}{l}\text { For } \alpha=4 \\
=24 \text { points }\end{array}$ & 12 points & 36 points \\
\hline$\sum_{u=1}^{N} x_{i u}^{2}$ & $2 \alpha\left(r^{2}+b^{2}\right)$ & $8 p^{2}$ & $2 \alpha\left(\mathrm{r}^{2}+\mathrm{b}^{2}\right)+8 \mathrm{p}^{2}$ \\
\hline$\sum_{u=1}^{N} x_{i u}^{4}$ & $\frac{\alpha r^{2}\left(3 r^{4}+4 b^{4}\right)}{2}$ & $8 p^{4}$ & $\begin{array}{l}\frac{1}{2} \alpha\left(3 r^{4}+4 b^{4}\right)+ \\
8 p^{4}\end{array}$ \\
\hline$\sum_{u=1}^{N} x_{i u}^{2} x_{j u}^{2}$ & $\frac{\alpha r^{2}\left(3 r^{4}+4 b^{4}\right)}{2}$ & $4 p^{4}$ & $\begin{array}{l}\frac{1}{4} \alpha r^{2}\left(r^{2}+8 b^{2}\right)+ \\
4 p^{4}\end{array}$ \\
\hline$\sum_{u=1}^{N} x_{i u}^{4}-3 \sum_{u=1}^{N} x_{i u}^{2} x_{j u}^{2}$ & $\frac{\left(3 r^{4}-24 r^{2} b^{2}+8 b^{4}\right) \alpha}{4}$ & $-4 p^{4}$ & $\begin{array}{l}3 r^{4}-24 r^{2} b^{2}+8 b^{4}- \\
4 p^{4}\end{array}$ \\
\hline
\end{tabular}




\section{A PRACTICAL HYPOTHETICAL EXAMPLE}

The design was set up to find out the effect of three fertilizer ingredients on the yield of solanum tuberosum in Nandi county to illustrate the application of the thirty six points second order rotatable design in three dimensions under field conditions.

The fertilizer ingredients and actual amount applied were;

a) Nitrogen(N) $x_{1} \gamma_{1}=30$ miligram per hole,

b) $\operatorname{Potash}\left(k_{2} o\right) x_{2} \gamma_{2}=25$ milligram per hole,

c) Phosphoric $\left(p_{2} O_{5}\right) x_{3} \gamma_{3}=35$ milligram per hole.

The average yield in $\mathrm{mg}$ per hole of the solanum tuberlosum was the response of interest in the experiment.

The original letter parameters shows the variation in the quantity of a factor applied due to the soil fertility gradient resulting in several radii manifestations of rotatability criterion.

According to Box [5] and Wilson [4], It can be recalled that the natural levels of these mineral elements denoted $\gamma_{i u}$ where Bose and Draper [9] scaling down condition fixes a particular design when $\lambda_{2}=1$, thus

$x_{i u}=\frac{\gamma_{i u}-\gamma_{i}}{s_{i}}$

$\gamma_{i}=\frac{\sum_{u=1}^{N} \gamma_{i u}}{N}$

$S_{i}=\left[\frac{\sum_{u=1}^{N}\left(\gamma_{i u}-\gamma_{i}\right)^{2}}{N}\right]^{0.5}$

$\gamma_{i u}=x_{i u} s_{i}+\gamma_{i u}$

For $\sum_{u=1}^{N} x_{i u}^{2}=\mathrm{N}$ and $\sum_{u=1}^{N} x_{i u}=0$

Evaluation of the inverse is the major computational challenge to estimate the coefficients of the second order rotatable design model which give the optimum yield. This requires a separate quest but the actual yields can be obtained if a field experiment is conducted as explained.

Let the scale parameter assumes $s_{1}=0.7, s_{2}=0.5$ and $s_{3}=1$ to estimate the co-efficient of a second order model in three factors, we require the field observations of the yield $y_{u}(u=1,2, \ldots, 36)$

The complete second order model to be fitted to yield values is,

$y_{u}=\beta_{o}+\sum_{i=1}^{36} \beta_{i} x_{i}+\sum_{u+1}^{36} \beta_{i i} x_{i}^{2}+\sum_{u=1}^{36} \beta_{i j} x_{i} x_{j}+e$

For the thirty six points second order rotatable design in three dimensions, we have the following coded and natural levels respectively as treatments in the table below.

Table 2. The coded values and natural levels in a practical field experiment.

\begin{tabular}{|c|c|c|c|c|c|}
\hline \multicolumn{2}{|l|}{ Coded levels } \\
\hline$x_{1 u}$ Natural levels & $x_{2 u}$ & $x_{3 u}$ & $\gamma_{1 u}$ & $\gamma_{2 u}$ & $\gamma_{3 u}$ \\
\hline 0.78 & 0 & 1.41 & 30.55 & 25.00 & 36.41 \\
\hline 0.55 & 0.55 & -1.41 & 30.39 & 25.28 & 33.59 \\
\hline 1.41 & 0.78 & 0 & 30.99 & 25.39 & 35.00 \\
\hline
\end{tabular}


International Journal of Advances in Scientific Research and Engineering (ijasre), Vol 5 (6), June-2019

\begin{tabular}{|c|c|c|c|c|c|}
\hline-1.41 & 0.55 & 0.55 & 29.01 & 25.28 & 35.55 \\
\hline 0 & 1.41 & 0.78 & 30.00 & 25.71 & 35.78 \\
\hline 0.55 & -1.41 & 0.55 & 30.39 & 24.30 & 35.55 \\
\hline 0 & 0.78 & 1.41 & 30.00 & 25.39 & 36.41 \\
\hline 0.55 & 0.55 & -1.41 & 30.39 & 25.28 & 33.59 \\
\hline 1.41 & 0 & 0.78 & 30.99 & 25.00 & 35.78 \\
\hline-1.41 & -0.55 & 0.55 & 29.01 & 24.62 & 35.55 \\
\hline 0.78 & 1.41 & 0 & 30.55 & 25.71 & 35.00 \\
\hline 0.55 & -1.41 & 0.55 & 30.39 & 24.30 & 35.55 \\
\hline-0.78 & 0 & 1.41 & 29.45 & 25.00 & 36.41 \\
\hline-0.55 & -0.55 & -1.41 & 29.62 & 24.62 & 33.59 \\
\hline 1.41 & -0.78 & 0 & 30.99 & 24.61 & 35.00 \\
\hline-1.41 & -0.55 & -0.55 & 29.01 & 24.62 & 34.45 \\
\hline 0 & 1.41 & -0.78 & 30.00 & 25.71 & 34.22 \\
\hline-0.55 & -1.41 & -0.55 & 29.62 & 24.30 & 34.45 \\
\hline 0 & -0.78 & 1.41 & 30.00 & 24.61 & 36.41 \\
\hline 0.55 & -0.55 & -1.41 & 30.39 & 24.62 & 33.59 \\
\hline 1.41 & 0 & -0.78 & 30.99 & 25.00 & 34.22 \\
\hline-1.41 & 0.55 & -0.55 & 29.01 & 25.28 & 34.45 \\
\hline-0.78 & 1.41 & 0 & 29.45 & 25.71 & 35.00 \\
\hline-0.55 & -1.41 & 0.55 & 29.62 & 24.30 & 35.55 \\
\hline 1 & 1 & 0 & 30.70 & 25.50 & 35.00 \\
\hline-1 & 1 & 0 & 29.30 & 25.50 & 35.00 \\
\hline 1 & -1 & 0 & 30.70 & 24.50 & 35.00 \\
\hline-1 & -1 & 0 & 29.30 & 24.50 & 35.00 \\
\hline 1 & 0 & 1 & 30.70 & 25.00 & 36.00 \\
\hline
\end{tabular}




\begin{tabular}{|c|c|c|c|c|c|}
\hline-1 & 0 & 1 & 29.30 & 25.00 & 36.00 \\
\hline 1 & 0 & -1 & 30.70 & 25.00 & 34.00 \\
\hline-1 & 0 & -1 & 29.30 & 25.00 & 34.00 \\
\hline 0 & 1 & 1 & 30.00 & 25.50 & 36.00 \\
\hline 0 & -1 & 1 & 30.00 & 24.50 & 36.00 \\
\hline 0 & 1 & -1 & 30.00 & 25.50 & 34.00 \\
\hline 0 & -1 & -1 & 30.00 & 24.50 & 34.00 \\
\hline
\end{tabular}

\section{APPLICATIONS}

This kind of investigations provides very useful information in research fields of chemical industry, agriculture and medicine.

The thirty six points design considered provides spherical information contours besides optimum combinations of treatments in industrial, medical and agricultural fields which results in economic use of scarce resources in relevant production processes.

It is important to note that the practical applications of these methods are not automatic, sometimes judgment is required. If an experimenter applies insufficient intellect to his results, he is likely to suffer as in any other method of experiment and it is likely to happen in new experiments where unfortunate selection of experimental units is made.

The second order rotatable design will be available when an experimenter wants to carry out an experiment where the responses will facilitate the estimation of the linear and quadratic co-efficient.

\section{CONCLUSIONS}

The study presents an illustration on how to obtain the mathematical parameters of the coded values and its corresponding natural levels of a second order rotatable design in three dimensions.

Response surface methodology in this case is used to approximate the functional relationship between the performance characteristics and the design variables.

After experimentation, the resulting response is used to construct response surface approximation model using least squares regression analysis.

\section{REFERENCES}

[1] A .Cheruiyot, Efficiencies of specific second order rotatable designs in three dimensions, M.Phil . Thesis, University of eldoret,Kenya, 2015

[2] N.O.Cornelious, Optimal sequential third order rotatable designs in three,four and five dimensions, M.Phil. Thesis,Moi University, Eldoret,Kenya,(2016)

[3] N.O. Cornelious, Construction of Thirty Nine Points Second Order Rotatable Design In Three Dimensions With A Practical Hypothetical Example. European International Journal of Science and Technology, 8 (2019), 51-57.

[4] N.O. Cornelious, A Hypothetical Sequential Third Order Rotatable Design in Five Dimensions. European International Journal of Science and Technology, 8(2019), 40-50.

[5] N.O. Cornelious, A Sequential Third Order Rotatable Design in Four Dimensions. Current Journal of Applied Science and Technology, (2019). 1-7.

[6] G.E. Box, Multifactor designs of first order,Journal of Biometrika 39(1952),49-57.

[7] G.E.P. Box and J.S. Hunter, Multifactor experimental designs for exploring response surfaces, Annals of mathematical statistics 28(1957). 195-241

[8] G.E.P.Box and K.B.Wilson, On experimental attainment of optimum conditions,Journal of Royal Statistical Society.Ser.13(1951),49-57

[9] J.K.Koske,J.M.Mutiso and I.K.Tum, Construction of a practical optimum second order rotatable design in three dimensions, Advances and Applications in Statistics 30(2012),31-43

[10] J.K.Koske,J.M.Mutiso and M.K.Kosgei, A specific optimum second order rotatable design of twenty four points with a practical example, Moi university,Eldoret,Kenya, 2008 
[11] J.M.Mutiso,Second and third order specific and sequential rotatable designs in k dimensions, D.Phil.Thesis,Moi University,Eldoret, Kenya, 1998

[12] R.C.Bose and Draper, second order rotatable designs in three dimensions, Annals of mathematical statistics 30(1959), 1097-1112

[13] S.J.Keny,I.K.Tum and E.K.Chirchir, Construction of thirty two points specific optimum second order rotatable design in three dimensions with a practical example, International journal of current research 4(2012),119-122 